\title{
AMNIOTIC FLUID EMBOLISM- PRESENTING AS GENERALISED ERYTHEMA AND RASHES (POSSIBILITY OF ANAPHYLACTOID REACTION SECONDARY TO AMNIOTIC FLUID EMBOLISM)
}

\author{
Priya Veluthakunju ${ }^{1}$ \\ ${ }^{1}$ Assistant Professor, Department of Obstetrics and Gynaecology, Government Medical College, Kottayam.
}

\section{ABSTRACT}

\section{BACKGROUND}

Amniotic fluid embolism is an obstetric emergency in which amniotic fluid, foetal hair cells enters maternal circulation causing cardiorespiratory collapse. This paper is reporting a rare case of amniotic fluid embolism, presenting as generalised oedema and rashes along with other clinical manifestations.

\section{KEYWORDS}

Amniotic Fluid Embolism, Peripheral Capillary Oxygen Saturation, Synchronised Intermittent Mandatory Ventilation, Neonatal Death, Maternal Mortality Rate.

HOW TO CITE THIS ARTICLE: Veluthakunju P. Amniotic fluid embolism - presenting as generalised erythema and rashes (possibility of anaphylactoid reaction secondary to amniotic fluid embolism). J. Evolution Med. Dent. Sci. 2017;6(13):1059-1060, DOI: $10.14260 /$ Jemds/2017/228

\section{BACKGROUND}

Amniotic fluid embolism is a rare but often fatal complication of pregnancy whose onset can neither be predicted nor prevented. Early diagnosis, expeditious resuscitation and delivery, management of sequelae by a team approach including obstetrician and intensivist may improve maternal and foetal outcome. Despite aggressive and early management, maternal and foetal morbidity and mortality remained unacceptably high.

\section{Case Report}

A 25-year-old primi gravida, who had regular antenatal check-up reported at 37 weeks 5 days to labour room at 8.30 AM. On examination, she was moderately built and nourished (Height: $145 \mathrm{~cm}$, Weight: $54 \mathrm{~kg}$, Body Mass Index: 25.71); vitals were normal. Per Abdomen: Uterus corresponds to term, cephalic presentation, foetal heart good with mild contraction. She was kept under observation in the labour room. At 3.45 PM, she developed leaking per vaginum. Per speculum examination showed leaking. Liquor was thick meconium stained. Per vaginal examination: Cervix soft, mid position, cervix $3 \mathrm{~cm}$ long, $1.5 \mathrm{~cm}$ dilated membrane absent, thick meconium stained liquor draining vertex at -3 . Pelvis was normal. Since liquor was thick meconium stained amnioinfusion given. Suddenly patient developed generalised itching, chest discomfort, erythema and urticarial rashes, first on the legs later became generalised. Patient had no history of atopy or drug reaction.

Sudden infusion of Inj. Hydrocortisone and antihistamines was given along with oxygen.

Patient developed drowsiness, bradycardia (Pulse Rate: $40 / \mathrm{mt}$ ), hypotension (Blood pressure less than $90 \mathrm{~mm}$ of

Financial or Other, Competing Interest: None.

Submission 06-01-2017, Peer Review 01-02-2017,

Acceptance 06-02-2017, Published 13-02-2017.

Corresponding Author:

Dr. Priya Veluthakunju,

\#1A, Meridian Symphony,

Manjelipadom Road,

North Fort Gate, Tripunithura,

Ernakulam-682301, Kerala, India.

E-mail: priya.drv@gmail.com

DOI: $10.14260 / \mathrm{jemds} / 2017 / 228$

mercury) and hypoxia ( $\mathrm{SPO}_{2}$ (peripheral capillary oxygen saturation) not recordable). This was followed by Cyanosis, dyspnoea, oedema lips and laryngeal oedema. Cardiopulmonary resuscitation started, Anaesthetist called in and patient was shifted to the theatre. Intubation was difficult due to laryngeal oedema. Lower Segment Caesarean Section under General Anaesthesia was done and delivered a female baby of $2.2 \mathrm{~kg}$ on 18.1.2014 at 4.15 PM 1 Apgar 2. Liquor was meconium stained. Since bleeding was slightly more than normal, uterine artery ligation done on both sides. Uterotonics were administered. Patient was shifted to Intensive Care Unit.

Antibiotics Inj. Piptaz and Inj. Metrogyl given; 1 pint Packed Cell and 5 pints Fresh Frozen Plasma was given in the post-operative period. In post-operative period, patient developed post-partum haemorrhage and was controlled by medical measures. Patient was put in SIMV (Synchronised Intermittent Mandatory Ventilation) mode. Patient was extubated after 5 days. Steroid dosage was tapered after 7 days. Baby NND (Neonatal Death) on 7th day. After 10 days, patient was discharged.

\section{Based on the findings of -}

1. Hypoxia (Pulse oxymetry saturation level less than 90\%).

2. Hypotension (Systolic blood pressure less than $90 \mathrm{~mm}$ of mercury).

3. Coagulation Parameters (PT-INR > 1.7).

Diagnosis of Amniotic Fluid Embolism is made. Rashes and erythema, which was generalised followed by lip oedema and laryngeal oedema may be due to anaphylactic reaction to Amniotic Fluid Embolism.

\section{DISCUSSION}

During pregnancy, intact membranes separate maternal circulation from amniotic fluid. In amniotic fluid embolism, amniotic fluid enters maternal circulation either with rupture of membranes, uterine trauma, small tears in lower uterine segment or during placental abruption at site of placental implantation. Typically, amniotic fluid embolism occurs during labour, delivery or in immediate post-partum period. 
Clinical Presentation can be Typical or Atypical[1]

Typical or classical form of amniotic fluid embolism is seen in 3 phases (Described by Clark et al in 1995).

Phase 1 - Respiratory and cardiovascular dysfunction.

Phase 2 - Coagulopathy.

Phase 3 - Acute respiratory distress syndrome.

Atypical presentation shows lack of cardiopulmonary collapse. Four classes of atypical presentation have been described -

1. Uterine haemorrhage type of atypical amniotic fluid embolism.

2. Acute respiratory distress syndrome type of atypical amniotic fluid embolism.

3. Paradoxical amniotic fluid embolism.

4. Caesarean section related atypical amniotic fluid embolism.

This patient has been administered with no other drugs other than saline infusion into uterine cavity. Also, this patient had no history of allergy to oxytocin.

\section{Pathophysiology}

Amniotic fluid embolism was thought to cause mechanical obstruction which leads to severe pulmonary hypertension, decreased (L) sided filling and consequently hypotension. Amniotic fluid embolism syndrome is that amniotic fluid that contains a complex mixture of maternal extracellular fluid, foetal urine, foetal squamous cells, lanugo hair, vernix caseosa, mucus, meconium, foetal antigens and potent vasoactive components such as thromboplastin, plasmin activator, vasoconstrictor endothelin, platelet activating factor, prostaglandin $\mathrm{F} 2 \infty$ (a potent pulmonary vasoconstrictor) and endogenous mediators like histamine, bradykinin, endothelin, leukotriene and arachidonic acid metabolites. Immune immunoglobulin IgE-mediated anaphylactic or non-immune non-IgE anaphylactoid reaction to amniotic fluid involve mast cell and basophil stimulation causing activation of complement, thrombin and coagulation cascade which are potentially fatal and require emergency management. Leukotriene in Amniotic Fluid are thought to cause localised or generalised anaphylactoid reaction, which lead to alveolar capillary leak and pulmonary oedema.
Amniotic fluid embolism result due to anaphylaxis of foetal material leaking into maternal circulation. Mast cell degranulation and elevated serum tryptase level has been reported in Amniotic Fluid Embolism. Serum tryptase level is the single most useful diagnostic test to confirm anaphylaxis. [2]

Review of literature (Acta Anaesthesia of Scand 2006 (50; 324-330) also describes six patients with cutaneous reactions (urticaria, generalised rash or angio-oedema) in patients with amniotic fluid embolism. [3]

\section{Maternal and Foetal Outcome Maternal Outcome}

In 1995, Clark reported MMR (Maternal Mortality Rate) of 61\%. In 2009, Matsuda reviewed care (1989 - 2000) in a tertiary care hospital and found to be $44.4 \%$. He also found that mean interval between clinical symptom and treatment was significantly shorter for survivors. The time interval to delivery following maternal arrest with best neonatal outcome in 5 - $15 \mathrm{mts}$.

\section{Foetal Outcome}

Perinatal outcome is $21 \%$. In 2010 , perinatal mortality rate is $135 / 1000$ births.

\section{Recurrence}

In 1992, Clark described successful pregnancy outcome of two pregnancies in women with previous amniotic fluid embolism. This patient also had uneventful second delivery.

\section{REFERENCES}

[1] Uszyński W, Uszyński M. Amniotic fluid embolism (AFE): a review of the literature orientated on two clinical presentations-typical and atypical. Open Journal of Obstetrics and Gynecology 2014;4:54-60.

[2] Clark SL, Hankins GVD, Dudley DA, et al. Amniotic fluid embolism: analysis of national registry. Am J Obste Gynecol 1995;172(4 Pt 1):1158-67.

[3] Harboe T, Benson MD, Oi H, et al. Cardiopulmonary distress during obstetrical anaesthesia: attempts to diagnose amniotic fluid embolism in a case series of suspected allergic anaphylaxis. Acta Anesthesia Scand 2006;50(3):324-30. 